\title{
Close correlation between CXCR4 and VEGF expression and their prognostic implications in nasopharyngeal carcinoma
}

\author{
YUICHI SEGAWA ${ }^{1,2}$, YOSHINAO ODA ${ }^{1}$, HIDETAKA YAMAMOTO ${ }^{1}$, HIDEKI SHIRATSUCHI $^{2}$, \\ NAOYA HIRAKAWA ${ }^{2}$, SHIZUO KOMUNE ${ }^{2}$ and MASAZUMI TSUNEYOSHI ${ }^{1}$ \\ Departments of ${ }^{1}$ Anatomic Pathology, and ${ }^{2}$ Otolaryngology, Graduate School of Medical Sciences, \\ Kyushu University, Fukuoka 812-8582, Japan
}

Received October 1, 2008; Accepted January 5, 2009

DOI: $10.3892 /$ or_00000341

\begin{abstract}
The CXCL12/CXCR4 pathway, which is involved in biological phenomena such as inflammation, lymphoid homing and regeneration, may play an important role in tumor progression and distant metastasis, especially in organselective metastasis. In addition, the CXCL12/CXCR4 pathway has been reported to regulate tumor angiogenesis. In this study, we examined the immunohistochemical expression of CXCR4 and vascular endothelial growth factor (VEGF) in nasopharyngeal carcinoma. CXCR4 and VEGF mRNAs were also assessed by real-time reverse transcription-polymerase chain reaction. Overexpression of CXCR4 and VEGF was observed in $41(53.9 \%)$ and $30(39.5 \%)$ of 76 tumors, respectively. There was a significant positive correlation between immunohistochemical CXCR4 and VEGF expression $(\mathrm{p}=0.0339)$. Additionally, immunohistochemical CXCR4 expression was associated with CXCR4 mRNA expression, and immunohistochemical VEGF expression was associated with VEGF mRNA expression $(\mathrm{p}=0.0040$ and $\mathrm{p}=0.0476$, respectively). Furthermore, patients with VEGF-positive tumors had a significantly worse prognosis than patients with VEGF-negative primary tumors $(\mathrm{p}=0.0044)$. Our findings suggest that the expression of CXCR4 and VEGF is associated with metastatic progression, and that VEGF expression is a valuable prognostic marker in nasopharyngeal carcinoma.
\end{abstract}

\section{Introduction}

Nasopharyngeal carcinoma (NPC) is an endemic disease with a distinct racial and geographic distribution. High-incidence areas of NPC are found in Southeast Asia and North Africa

Correspondence to: Dr Yoshinao Oda, Department of Anatomic Pathology, Graduate School of Medical Sciences, Kyushu University, Maidashi 3-1-1, Higashi-ku, Fukuoka 812-8582, Japan

E-mail: oda@surgpath.med.kyushu-u.ac.jp

Key words: CXCR4, VEGF, nasopharyngeal carcinoma and low-incidence areas are in the USA, Europe and Japan. NPC is divided into two histological subtypes according to the World Health Organization (WHO) classification of 2005: i) keratinizing squamous cell carcinoma and nonkeratinizing carcinoma. Death from NPC is usually due to distant metastasis, especially in patients with non-keratinizing carcinoma; ii) distant metastases of NPC are more frequent than those of other malignant tumors of the head and neck; and iii) NPC shows a preference for the bone, lung and liver, and its metastatic sites and distant metastasis correlate significantly with poor survival. The elucidation of the metastatic mechanism may lead to the development of successful therapies for NPC.

Chemotaxis is an important mechanism involved in biological phenomena such as inflammation, lymphoid homing and regeneration. It has been demonstrated that abnormal chemotaxis can participate in cancer metastasis. Since Müller et al (4) first identified a chemotaxis-related factor, finding that chemokines and chemokine receptors play a critical role in determining the metastatic destination of tumor cells, several studies have indicated that tumor cells express chemokine receptors and may employ chemokinemediated mechanisms for organ specificity of metastasis (5-7). Additionally, recent studies have shown that the CXCL12/CXCR4 pathway may play an important role in pulmonary and bone metastases $(8,9)$. Angiogenesis is also a key step in tumor invasiveness and metastasis. Vascular endothelial growth factor (VEGF), a major angiogenetic factor, is overexpressed in several types of cancer and is known to participate in tumor metastasis. There is increasing evidence that the CXCL12/CXCR4 pathway regulates VEGF expression $(10,11)$ and that VEGF regulates CXCR4 expression $(12,13)$. Although several studies have demonstrated CXCR4 expression in NPC (14-17), the mechanisms that control NPC metastasis remain poorly understood. The purpose of the present study was to evaluate CXCR4 and VEGF expression in NPC.

\section{Materials and methods}

Patients. A total of 76 primary NPC biopsy specimens were obtained prior to treatment from patients at the Department of Otolaryngology of Kyushu University Hospital, the 
Department of Head and Neck Surgery of the National Kyushu Cancer Center, and the Department of Otolaryngology of the National Kyushu Medical Center between 1985 and 2007. The expressions of inducible nitric oxide synthase (iNOS), 8-hydroxydeoxyguanosine (8-OHdG), latent membrane protein 1 (LMP-1) and Epstein-Barr virusencoded small RNA (EBER) in 73 of these 76 patients have been published previously (18). Materials from all cases were fixed in a $10 \%$ formaldehyde solution and embedded in paraffin. In addition, snap-frozen samples from 14 primary tumors were obtained. The clinical stage and histological tumor type were determined according to the International Union Against Cancer (UICC) Classification of 1997 (fifth edition) and the WHO Classification of 2005 (1), respectively. $\mathrm{T}$ classification was divided into the low $\mathrm{T}$ ( $\mathrm{T} 1$ or T2) and high $\mathrm{T}$ ( T3 or T4) categories. $\mathrm{N}$ classification was divided into the lymph node-negative (N0) and lymph nodepositive (N1, N2 or N3) categories. Stage grouping was divided into the early stage (stage I or stage II) and the advanced stage (stage III or stage IV) (19). Eleven samples of nasopharyngeal tissues containing a non-malignant respiratory epithelium and lymphocytes without pathological evidence of cancer cells were retained as control samples.

Immunohistochemical examination and evaluation. Immunohistochemical staining was carried out using antibodies to CXCR4 (12G5, monoclonal; BD PharMingen, San Diego, CA, USA) and VEGF (A-20, polyclonal; Santa Cruz Biotechnology, Santa Cruz, CA, USA). Sections $(4-\mu \mathrm{m})$ thick were deparaffinized and dehydrated with xylene and ethanol. Antigen retrieval was performed by microwave heating in $10 \mathrm{mM}$ sodium citrate buffer ( $\mathrm{pH}$ 6.0) for $20 \mathrm{~min}$ for CXCR4, and in $10 \mathrm{mM}$ sodium citrate buffer with Tween $(\mathrm{pH} 6.0)$ for $30 \mathrm{~min}$ for VEGF. Endogenous peroxidase activity was blocked by 30 min of incubation with $0.3 \%$ hydrogen peroxidase in absolute methanol. Background staining was minimized by incubation with $1 \%$ normal rabbit serum for CXCR4 and with $1 \%$ normal goat serum for VEGF for $10 \mathrm{~min}$. Sections were incubated with a primary antibody overnight at $4^{\circ} \mathrm{C}$, followed by testing using a streptavidin-biotinperoxidase kit (Nichirei, Tokyo, Japan). Diaminobenzidine tetrahydrochloride was used as the chromogen. Finally, sections were counterstained with Mayer's hematoxylin. In each experiment, negative control sections were treated similarly with phosphate-buffered saline (PBS) instead of the primary antibody. Tissues from breast carcinoma and colon carcinoma were used as positive controls for CXCR4 and VEGF, respectively $(4,20)$.

Two pathologists (Y.S. and M.T.) evaluated all specimens and were blinded to clinical data and other immunohistochemical results. As previously described (21), slides were scored based on the strength of CXCR4 and VEGF expression: 0 , negative; $1+$, weak staining; $2+$, moderate staining; and $3+$, strong staining. The distribution of positive cells was also recorded in order to note the diffuse or focal nature of the positive cells: sporadic (positive cells $<10 \%)$, focal $(\geq 11 \%$ but $<50 \%$ ), and diffuse (positive cells $\geq 50 \%$ ). Immunohistochemical scores of $2+$ or $3+$ with a focal or diffuse distribution were considered to be positive for CXCR4 and VEGF antibodies.
RNA extraction and TaqMan real-time reverse transcriptionpolymerase chain reaction $(R T-P C R)$. Total RNA was extracted with $1 \mathrm{ml}$ of TRIzol reagent (Invitrogen, Carlsbad, CA, USA) from 14 snap-frozen tumor samples and the human breast cancer cell line MCF7, which was used as an external control, following the manufacturer's instructions. RNA (5 $\mu \mathrm{g}$ ) (from each sample was used for the subsequent reverse transcription. After the reaction, RNase treatment was performed to eliminate RNA.

Semi-quantitative PCR for CXCR4 and VEGF was performed and the results were analyzed using pre-developed TaqMan assay reagents (CXCR4, TaqMan Assay ID: Hs00237052_m1; VEGF, ID: Hs00173626_m1; glyceraldehydes-3-phosphate dehydrogenase (GAPDH), ID: Hs99999905_m1), and an ABI PRISM 7700 Sequence Detection System (Applied Biosystems, Foster City, CA, USA). The PCR reaction was carried out according to the manufacturer's protocol. According to the manufacturer, the pre-developed TaqMan assay reagents had equivalent PCR efficiency. The obtained data were thus normalized to an endogenous reference, GAPDH, using the comparative threshold cycle (CT) method according to the manufacturer's protocol.

Follow-up and statistical analysis. Fisher's exact test was used to evaluate the association between two dichotomous variables. Comparisons between two dichotomous variables with respect to CXCR4 or VEGF mRNA were performed using the Mann-Whitney U test. To determine the prognostic factor, we examined the outcomes of our 76 patients by reviewing their medical charts. The median follow-up term was 28.9 months (range, 1-128 months). Fifty-one patients had a follow-up period at least 2 years after treatment. We analyzed the overall survival of patients with at least a 2-year follow-up with respect to the immunohistochemical expression of CXCR4 and VEGF. The overall survival rate was estimated by the Kaplan-Meier method and compared by log-rank test. A p-value $<0.05$ was considered significant.

\section{Results}

Clinicopathological parameters. Our subjects consisted of 53 males and 23 females, ranging in age from 19 to 85 years (mean, 56.6 years). With respect to therapy, 2 patients underwent resection of the primary tumor and 15 patients underwent neck dissection. All patients underwent either radiotherapy $(n=73)$ or chemotherapy $(n=68)$, or both $(n=67)$. The types of anticancer drugs varied by institution, but combinations of carboplatin, cisplatin, peplomycin, bleomycin, and 5-fluorouracil were used. According to the UICC classification, 34 patients were assigned to the low $\mathrm{T}$ category (T1, 17 cases; T2, 17 cases) and 42 to the high T category (T3, 16 cases; T4, 26 cases). Thirteen patients were classified as lymph node-negative and 63 as lymph node-positive (N1, 16 cases; N2, 34 cases; N3, 13 cases). Additionally, 13 patients were assigned to the early stage category (stage I, 3 cases; stage II, 10 cases) and 63 to the advanced stage category (stage III, 23 cases; stage IV, 40 cases). Four patients (5.3\%) had distant metastases, one in the lung, one in the bone, one in the lung and bone, and one in the liver and bone. A review 

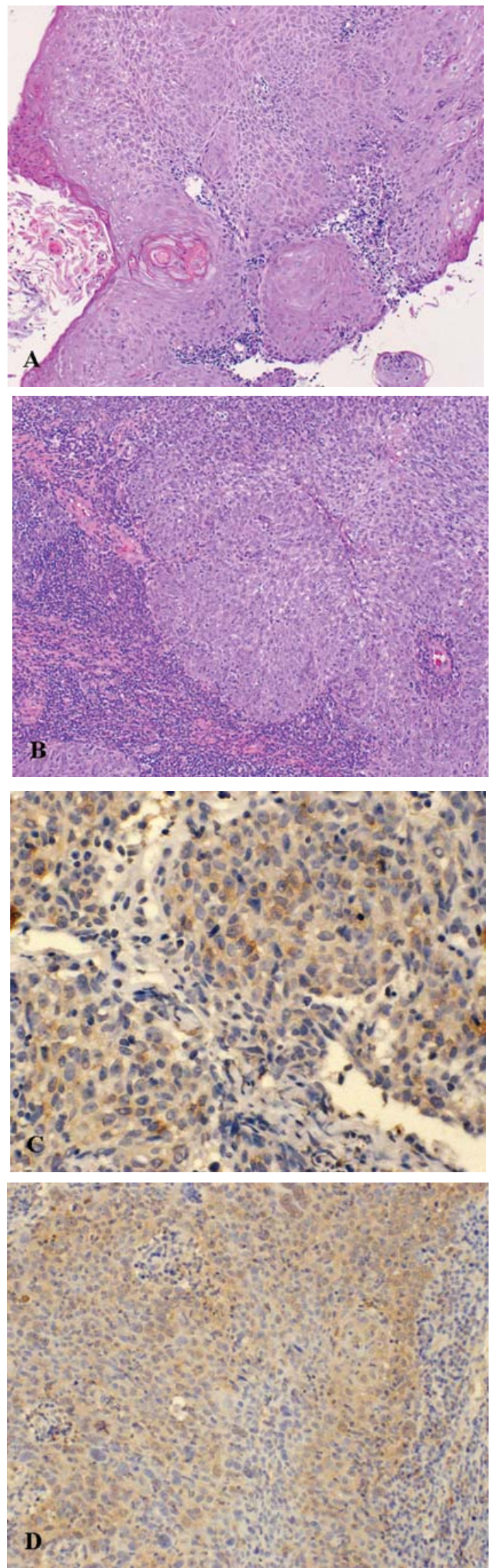

Figure 1. (A) Histological appearance of keratinizing squamous cell carcinoma composed of sheets of atypical squamous cells with keratinization. (B) Histological appearance of non-keratinizing carcinoma composed of oval carcinoma cells mingled with lymphocytes. (C) NPC revealing cytoplasmic and nuclear staining for CXCR4. (D) NPC revealing cytoplasmic staining for VEGF.
Table I. Immunohistochemical expression of CXCR4 and VEGF according to clinicopathological features.

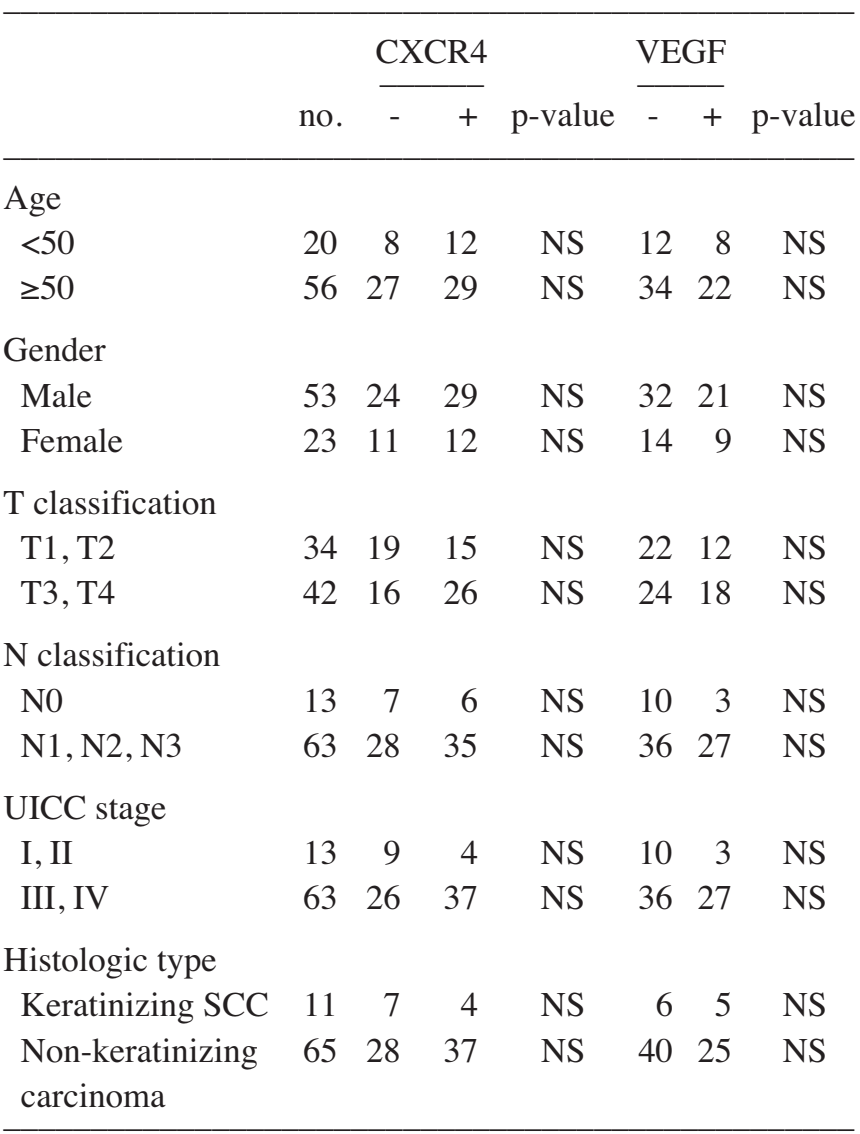

UICC, International Union Against Cancer; SCC, squamous cell carcinoma; NS, not significant.

of hematoxylin and eosin sections revealed 11 keratinizing squamous cell carcinomas (Fig. 1A) and 65 non-keratinizing carcinomas (Fig. 1B).

Immunohistochemical analyses of CXCR4 and VEGF. Fortyone $(53.9 \%)$ of 76 tumors showed cytoplasmic and nuclear staining for CXCR4 (Fig. 1C); the remaining 35 (46.1\%) were negative. No significant association was detected between CXCR4 expression and clinicopathological features (Table I). With respect to VEGF expression, the staining was predominantly confined to the cytoplasm (Fig. 1D). Thirty (39.5\%) of 76 tumors were positive for VEGF. No significant association was detected between VEGF expression and other clinicopathological features (Table II). VEGF expression was found to be significantly related to CXCR4 expression ( $\mathrm{p}=0.0339$, Table II). With respect to the 4 tumors with distant metastases, 3 with bone metastases exhibited both CXCR4 and VEGF expression, and the one with pulmonary metastasis showed no expression of either CXCR4 or VEGF. Furthermore, no CXCR4 or VEGF expression was observed in 11 samples of non-neoplastic nasopharyngeal epithelium.

Semi-quantitative assay for CXCR4 and VEGF $m R N A$ by TaqMan PCR. CXCR4 and VEGF mRNA levels were evaluated in tumors from 14 patients. The median $(25 \%$, 
Table II. Correlation between CXCR4 and VEGF immunohistochemical expression.

\begin{tabular}{llrrr}
\hline & & \multicolumn{2}{c}{ VEGF } & \\
\cline { 3 - 4 } & no. & - & + & p-value \\
\hline CXCR4 & & & & \\
- & 35 & 26 & 9 & $0.0339^{\mathrm{a}}$ \\
+ & 41 & 20 & 21 & $0.0339^{\mathrm{a}}$ \\
\hline
\end{tabular}

aStatistically significant.

$75 \%)$ CXCR4 mRNA level of NPC was found to be 4.710 $(2.278,8.570)$ and the relationship between CXCR4 mRNA level and immunohistochemical CXCR4 expression was analyzed. The median $(25 \%, 75 \%)$ of the CXCR4 mRNA level of CXCR4-negative tumors was $2.278(1.818,2.546)$ and that of CXCR4-positive tumors was $8.570(6.059,11.175)$. The CXCR4 mRNA level was found to be significantly correlated with CXCR4 immunohistochemical expression ( $\mathrm{p}=0.0040$, Fig. 2A). With respect to VEGF mRNA level, the median $(25 \%, 75 \%)$ was found to be $4.028(2.778,6.774)$, with a median $(25 \%, 75 \%)$ of VEGF-negative tumors of $2.778(1.476,3.916)$ and a median of VEGF-positive tumors of $5.987(4.316,7.071)$. The VEGF mRNA level was found to be significantly correlated with VEGF immunohistochemical expression ( $p=0.0476$, Fig. 2B).

Survival analysis. Patients with VEGF-positive tumors had a significantly shorter survival time than those with VEGFnegative tumors $(\mathrm{p}=0.0044$, Fig. 3A). CXCR4 expression also seemed to be associated with poor survival, but this association did not reach statistical significance $(p=0.2947$, Fig. 3B).
A

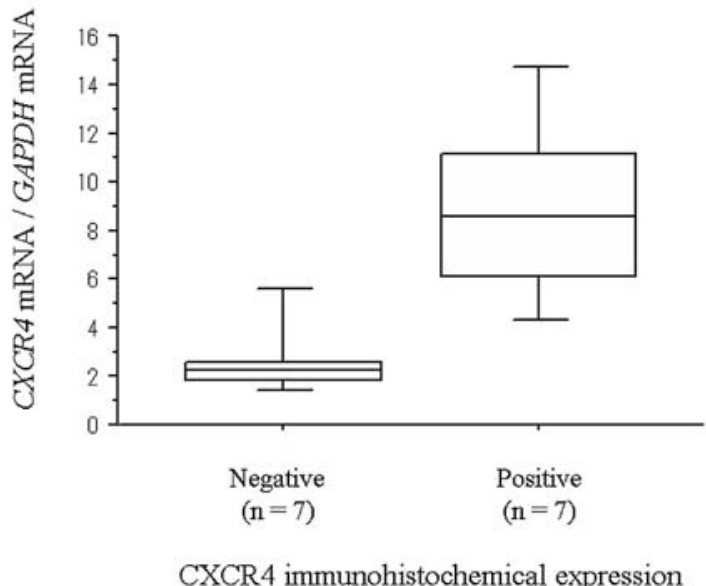

B

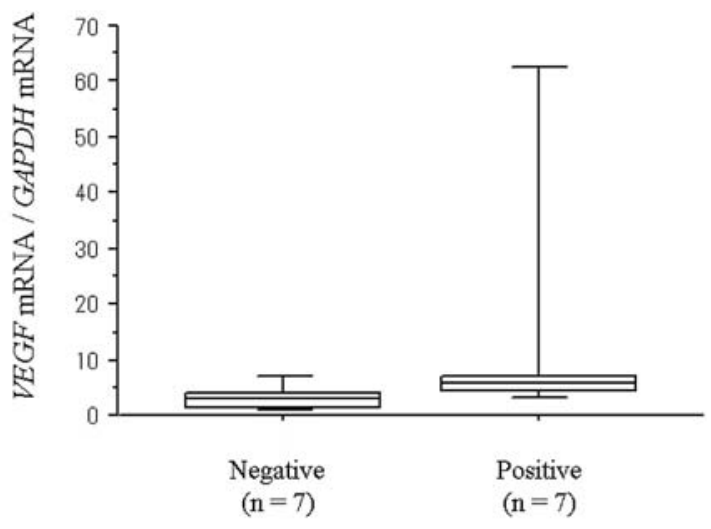

VEGF immunohistochemical expression

Figure 2. (A) Relationship between CXCR4 mRNA expression and CXCR4 immunohistochemical expression status in 14 cases. The boxes indicate the 75th and 25th percentiles, the horizontal line indicates the median, and the bars indicate the 10th and 90th percentiles. CXCR4 immunohistochemical expression status was significantly correlated with CXCR4 mRNA expression (p=0.0040, Mann-Whitney U test). (B) Relationship between VEGF mRNA expression and VEGF immunohistochemical expression status in 14 cases. VEGF immunohistochemical expression status was significantly correlated with VEGF mRNA expression ( $\mathrm{p}=0.0476$, Mann-Whitney U test).

A

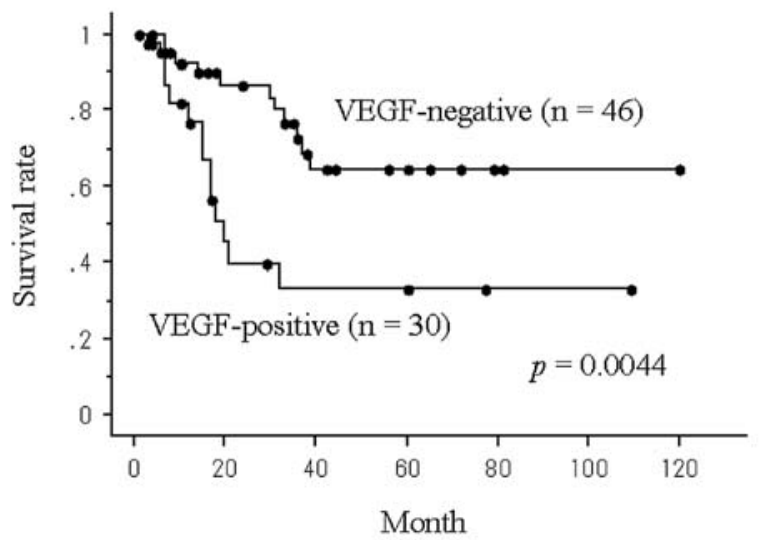

B

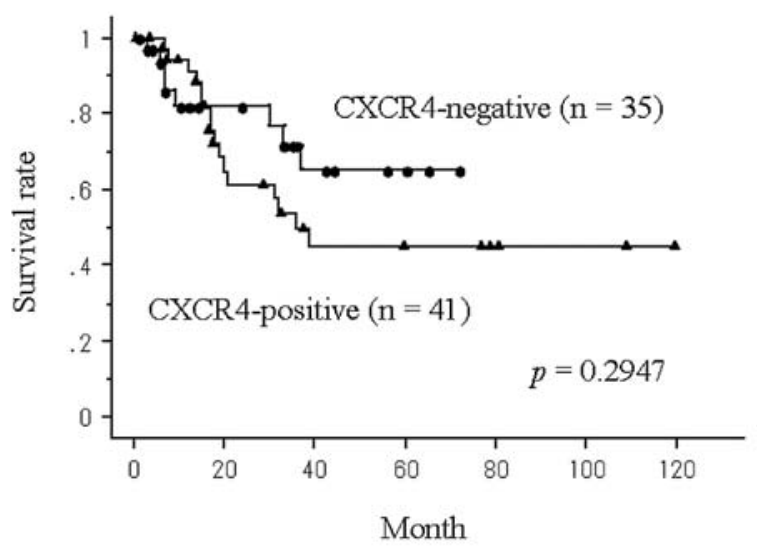

Figure 3. Kaplan-Meier analyses for overall survival. (A) The survival rate of patients with VEGF-positive tumors was significantly worse than that of patients with VEGF-negative tumors ( $\mathrm{p}=0.0044)$. (B) Patients with CXCR4-positive tumors showed a somewhat adverse prognosis compared with those with CXCR4-negative tumors, however, the present results were not -statistically significant $(\mathrm{p}=0.2947)$. 


\section{Discussion}

In the present study, we compared CXCR4 expression with VEGF expression in NPC, finding that CXCR4 expression was significantly correlated with VEGF expression in clinical samples of NPC. Several studies have revealed a relationship between the CXCL12/CXCR4 pathway and VEGF in carcinoma. In breast cancer, Bachelder et al (12) demonstrated that VEGF regulates CXCR4 expression in vitro, and Liang et al (11) showed that CXCR4 upregulates the phosphorylation of Akt and increases VEGF expression at both the mRNA and protein levels. In addition, CXCL12 has been shown to induce VEGF secretion in prostate (22), ovarian (13) and colorectal cancer (23). To the best of our knowledge, the present study is the first investigation revealing a close relationship between CXCR4 and VEGF expression in NPC.

CXCR4 plays an important role in promoting organselective metastasis. Murakami et al (24) demonstrated that CXCR4 expression in B16/F1 melanoma cells increases pulmonary metastasis ( $>10$-fold) without affecting metastasis to several other sites, including the lymph nodes, liver and kidney. Kang et al (9) showed that CXCR4 was overexpressed by subpopulations from a human breast cancer cell line, MDA-MB-231, with enhanced metastatic abilities to bone. These studies indicate that the CXCL12/CXCR4 pathway may play an important role in pulmonary and bone metastases. In the present study, 3 of 4 tumors, that developed bone metastasis stained positive for CXCR4. Further studies analyzing larger numbers of patients with NPC are necessary to elucidate organ-selective metastasis in this disease.

Distant metastases are found in about $5-7 \%$ of NPC patients at diagnosis. However, within the first 3 years following treatment, the overall incidence of metastases is approximately $25-30 \%$ (2). Prevention and treatment for distant metastasis is therefore an essential part of therapy for NPC. As noted above, the CXCL12/CXCR4 pathway may play an important role in the spread, progression and metastasis of many different types of tumors $(4,10,13,21)$ including NPC (14-17). CXCR4 antagonists may inhibit tumor progression and metastasis and are being evaluated in preclinical studies on the treatment of neoplastic tumors. Smith et al (25) found that inhibiting CXCR4 with specific antagonist AMD3100 delayed the growth of a highly metastatic mammary cell line, 4T1 cells, in the lung while Tamamura et al (26) showed that T140 analogs, which are CXCR4 antagonists, inhibited the stromal cell-derived factor (SDF)-1-induced migration of human MDA-MB-231 breast cancer cells. In addition, Hu et al (14) demonstrated that antisense CXCR4 inhibits the incidence of lung and lymph node metastasis of the NPC cell line in nude mice. These studies support the clinical use of CXCR4 antagonists in future NPC therapies.

VEGF plays a critical role in tumor growth and neovascularization. It has been demonstrated that increased VEGF expression is associated with tumor growth and metastasis, while inhibition of VEGF expression results in the suppression of tumor growth and tumor-induced angiogenesis (27). In NPC, VEGF has been reported to be up-regulated by the Epstein-Barr virus oncoprotein, LMP-1,
(28) and VEGF expression is known to have a significant association with angiogenesis measured in terms of microvessel density, lymph node metastases, recurrence and distant metastases $(29,30)$. In addition, several reports have shown that VEGF expression is a valuable prognostic marker for prognosis prediction in NPC, especially in advanced NPC $(31,32)$. In the present study, we also found that VEGF expression in NPC specimens was significantly related to shorter survival time. These previously reported observations together with the present results support the potential use of VEGF expression as a predictive marker of recurrence and metastasis in NPC.

In conclusion, the present study revealed the close relationship between CXCR4 and VEGF expression in NPC. Although CXCR4 expression was not a statistically significant prognostic factor in the current study, patients with CXCR4 expression seemed to have a poor prognosis. Therefore, CXCR4 may play an important role in promoting organselective metastasis. VEGF expression, on the other hand, was found to be a valuable prognostic factor in NPC. There is also the possibility that CXCR4 and VEGF antagonists may prevent tumor progression and distant metastases in NPC.

\section{Acknowledgements}

The authors would like to thank Ms. Y. Nouzuka and Ms. $\mathrm{N}$. Tateishi for their technical assistance. This work was supported in part by a Grant-in-Aid for Scientific Research (C) (18590332) from the Japan Society for the Promotion of Science. We thank KN International for revising the English used in this article.

\section{References}

1. Chan JKC, Bray F, McCarron P, et al: Nasopharyngeal carcinoma. In: WHO Classification of Tumours: Pathology and Genetics: Head and Neck Tumours. Barnes L, Eveson JW, Reichart P and Sidransky D (eds). IARC Press, Lyon pp85-97, 2005.

2. Licitra L, Bernier J, Cvitkovic E, et al: Cancer of the nasopharynx. Crit Rev Oncol Hematol 45: 199-213, 2003

3. Ahmad A and Stefani S: Distant metastases of nasopharyngeal carcinoma: a study of 256 male patients. J Surg Oncol 33: 194-197, 1986

4. Müller A, Homey B, Soto H, et al: Involvement of chemokine receptors in breast cancer metastasis. Nature 410: 50-56, 2001.

5. Muller A, Sonkoly E, Eulert C, et al: Chemokine receptors in head and neck cancer: association with metastatic spread and regulation during chemotherapy. Int J Cancer 118: 2147-2157, 2006.

6. Dellacasagrande J, Schreurs OJ, Hofgaard PO, et al: Liver metastasis of cancer facilitated by chemokine receptor CCR6. Scand J Immunol 57: 534-544, 2003.

7. Letsch A, Keilholz U, Schadendorf D, et al: Functional CCR9 expression is associated with small intestinal metastasis. J Invest Dermatol 122: 685-690, 2004.

8. Murakami T, Cardones AR and Hwang ST: Chemokine receptors and melanoma metastasis. J Dermatol Sci 36: 71-78, 2004.

9. Kang Y, Siegel PM, Shu W, et al: A multigenic program mediating breast cancer metastasis to bone. Cancer Cell 3: 537-549, 2003.

10. Bian XW, Yang SX, Chen JH, et al: Preferential expression of chemokine receptor CXCR4 by highly malignant human gliomas and its association with poor patient survival. Neurosurgery 61: 570-579, 2007. 
11. Liang Z, Brooks J, Willard M, et al: CXCR4/CXCL12 axis promotes VEGF-mediated tumor angiogenesis through Akt signaling pathway. Biochem Biophys Res Commun 359: 716-722, 2007.

12. Bachelder RE, Wendt MA and Mercurio AM: Vascular endothelial growth factor promotes breast carcinoma invasion in an autocrine manner by regulating the chemokine receptor CXCR4. Cancer Res 62: 7203-7206, 2002.

13. Kryczek I, Lange A, Mottram P, et al: CXCL12 and vascular endothelial growth factor synergistically induce neoangiogenesis in human ovarian cancers. Cancer Res 65: 465-472, 2005.

14. Hu J, Deng X, Bian X, et al: The expression of functional chemokine receptor CXCR4 is associated with the metastatic potential of human nasopharyngeal carcinoma. Clin Cancer Res 11: 4658-4665, 2005.

15. Ou DL, Chen CL, Lin SB, Hsu CH and Lin LI: Chemokine receptor expression profiles in nasopharyngeal carcinoma and their association with metastasis and radiotherapy. J Pathol 210: 363-373, 2006

16. Wang N, Wu QL, Fang Y, et al: Expression of chemokine receptor CXCR4 in nasopharyngeal carcinoma: pattern of expression and correlation with clinical outcome. J Transl Med 3: 26,2005

17. Li J, Zhang XS, Xie D, et al: Expression of immune-related molecules in primary EBV positive Chinese nasopharyngeal carcinoma: associated with latent membrane protein 1 (LMP1) expression. Cancer Biol Ther 6: 1997-2004, 2007.

18. Segawa Y, Oda Y, Yamamoto H, et al: Overexpression of inducible nitric oxide synthase and accumulation of $8-\mathrm{OHdG}$ in nasopharyngeal carcinoma. Histopathology 52: 213-223, 2008.

19. Horikawa T, Sheen TS, Takeshita H, Sato H, Furukawa M and Yoshizaki T: Induction of c-Met proto-oncogene by EpsteinBarr virus latent membrane protein-1 and the correlation with cervical lymph node metastasis of nasopharyngeal carcinoma. Am J Pathol 159: 27-33, 2001.

20. Takahashi Y, Kitadai Y, Bucana CD, Cleary KR and Ellis LM: Expression of vascular endothelial growth factor and its receptor, KDR, correlates with vascularity, metastasis, and proliferation of human colon cancer. Cancer Res 55: 3964-3968, 1995.

21. Oda Y, Yamamoto H, Tamiya S, et al: CXCR4 and VEGF expression in the primary site and the metastatic site of human osteosarcoma: analysis within a group of patients, all of whom developed lung metastasis. Mod Pathol 19: 738-745, 2006.
22. Darash-Yahana M, Pikarsky E, Abramovitch R, et al: Role of high expression levels of CXCR4 in tumor growth, vascularization and metastasis. FASEB J 18: 1240-1242, 2004.

23. Ottaiano A, Franco R, Aiello Talamanca A, et al: Overexpression of both CXC chemokine receptor 4 and vascular endothelial growth factor proteins predicts early distant relapse in stage II-III colorectal cancer patients. Clin Cancer Res 12: 2795-2803, 2006.

24. Murakami T, Maki W, Cardones AR, et al: Expression of CXC chemokine receptor- 4 enhances the pulmonary metastatic potential of murine B16 melanoma cells. Cancer Res 62: 7328-7334, 2002.

25. Smith MC, Luker KE, Garbow JR, et al: CXCR4 regulates growth of both primary and metastatic breast cancer. Cancer Res 64: 8604-8612, 2004.

26. Tamamura H, Hori A, Kanzaki N, et al: T140 analogs as CXCR4 antagonists identified as anti-metastatic agents in the treatment of breast cancer. FEBS Lett 550: 79-83, 2003.

27. Ferrara N: VEGF and the quest for tumour angiogenesis factors. Nat Rev Cancer 2: 795-803, 2002.

28. Murono S, Inoue H, Tanabe T, et al: Induction of cyclooxygenase- 2 by Epstein-Barr virus latent membrane protein 1 is involved in vascular endothelial growth factor production in nasopharyngeal carcinoma cells. Proc Natl Acad Sci USA 98: 6905-6910, 2001.

29. Wakisaka N, Wen QH, Yoshizaki T, et al: Association of vascular endothelial growth factor expression with angiogenesis and lymph node metastasis in nasopharyngeal carcinoma. Laryngoscope 109: 810-814, 1999.

30. Guang-Wu H, Sunagawa M, Jie-En L, et al: The relationship between microvessel density, the expression of vascular endothelial growth factor (VEGF), and the extension of nasopharyngeal carcinoma. Laryngoscope 110: 2066-2069, 2000.

31. Li YH, Hu CF, Shao Q, et al: Elevated expressions of survivin and VEGF protein are strong independent predictors of survival in advanced nasopharyngeal carcinoma. J Transl Med 6: 1, 2008.

32. Krishna SM, James S and Balaram P: Expression of VEGF as prognosticator in primary nasopharyngeal cancer and its relation to EBV status. Virus Res 115: 85-90, 2006. 